\title{
INSIDE THE USCAP JOURNALS
}

\section{MODERN PATHOLOGY}

\section{Pediatric lymphoma mutational landscape}

See page 1212

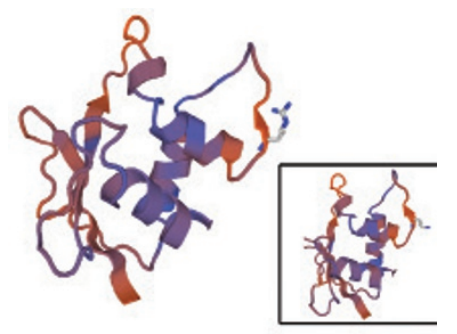

Ozawa et al compared the distinct features of two rare forms of pediatric B-cell lymphomas with their adult counterparts. They performed a detailed whole-exome deep sequencing analysis of a cohort of pediatric-type follicular lymphoma and pediatric marginal-zone lymphomas. A recurrent somatic variant encoding p.Lys66 Arg in the transcription factor interferon regulatory factor 8 (IRF8) in three of six cases was present in the pediatric-type follicular lymphoma. Additional somatic point mutations were identified in genes involved in transcription, intracellular signaling, and cell proliferation. Translocations involving BCL2 were not detected by exome sequencing; however, one case of pediatric nodal marginalzone lymphoma was shown to have a synonymous mutation in angiomotin like-1 (AMOTL1), a specific point mutation previously identified in a patient with carcinoma of the large intestine. The mutation observed in IRF8 in B-cell lymphoma is predicted to have a deleterious effect and has been identified in a subset of patients in whom it could be a novel driver, promoting disease development.

\section{Harmonization of PD-L1 immunohistochemistry See page 1165}

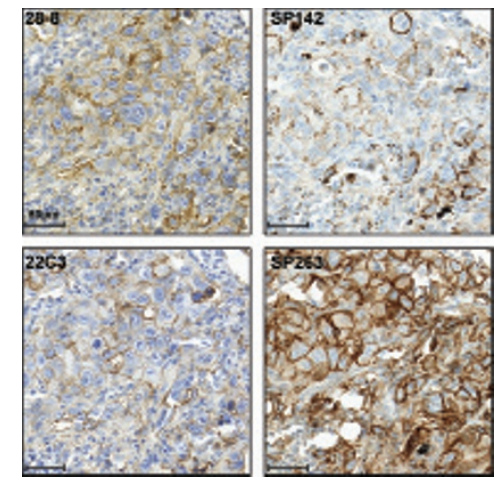

Anti-PD-1 and anti-PD-L1 immunotherapy is a growing and increasingly successful anticancer therapeutic approach. Immunohistochemistry assays for the PD-L1 protein can enhance clinical decision making, and Scheel and colleagues sought to assess the reliability of such assays. Two sets of samples, each with 15 resection specimens, were centrally stained for PD-L1 and scored by nine pathologists. The results were reproducible for two laboratory-based assays and four clinical trial assays. The group developed an integrative scoring system because studies have shown that response rates to immunotherapy can be related to PD-L1-positive carcinoma cells. The four clinical trial assays elicited no significant differences in interobserver levels despite their known different staining patterns, a point that the authors found intriguing. Further development of assays to assess PD-1 and PD-L1 expression in cancer will add to their predictive value and further elucidate the relationship between PD-L1 expression and patient response to immunotherapy.

\section{LABORATORY INVESTIGATION}

\section{Immortalizing neurofibroma cells}

See page 1105

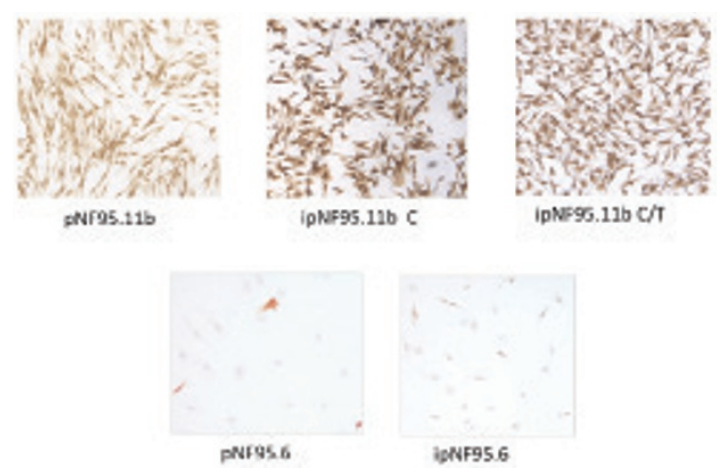

Li et al investigated the development and treatment of neurofibromas, known to be associated with biallelic loss of NF1 gene function. The group sought to reduce the difficulty of deriving enriched human Schwann cell cultures, which enter replicative senescence after 6-10 passages. They developed cell culture methods by creating immortalized Schwann cells using lentiviral transduction of $C d k 4$ and telomerase. The immortalized cells provide increased purity, greater expansion capacity, and advantageous culture conditions. These cell lines have been used in assays to elucidate neurofibroma development, in tumor investigations, and in genetic manipulation to create 
new models and rescue experiments. Li and colleagues developed paired immortalized stem cell lines from normal nerve and plexiform neurofibroma from the same patient, providing the opportunity to perform experiments in which an isogenic background is helpful.

\section{Sinomenine alleviates arthritis by AhR activation}

See page 1076

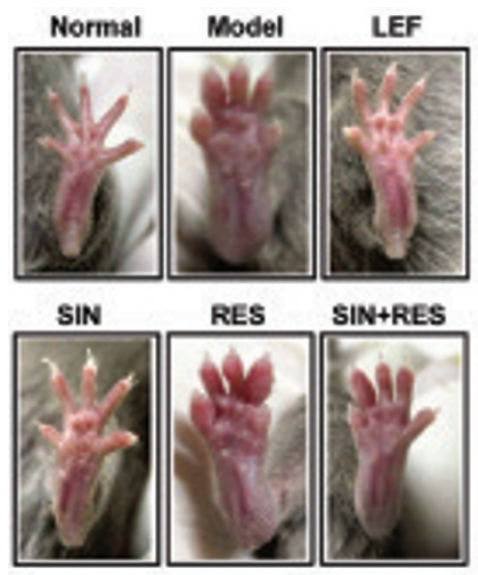

An increasing body of evidence suggests that sinomenine (SIN), a plant-derived isoquinoline alkaloid, has efficacy for rheumatoid arthritis. It has been used for this purpose for several decades in China. Aryl hydrocarbon receptor (AhR) is a ligand-dependent transcription factor that mediates the biotransformation and carcinogenic/teratogenic effects of environmental toxins. AhR is expressed in barrier tissues by immune cells, and in the gut it can find ligands in the microflora, inducing nuclear translocation and transcriptional activation. Using a computer model, Tong et al found that SIN acted as an AhR ligand and increased CYP1A1 expression and activity. This was dependent on de novo RNA synthesis and protein synthesis. As an AhR agonist, SIN induced T regulatory cell generation and function, thus alleviating arthritis. The authors maintain that this places SIN in a category of drugs that have potential to act as first-line therapies in autoimmune diseases.

\section{nature.com/pathology}

\section{Reward increases immunity}

Ben-Shaanan et al sought to explore the activation of the reward system and its effect on the immune system. Using DREADDs (designed receptors exclusively activated by designed drugs), they directly activated dopaminergic neurons in the mouse ventral tegmental area (VTA). With subsequent

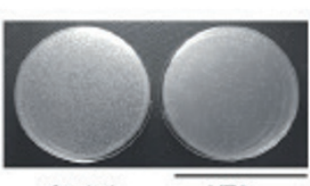

Control

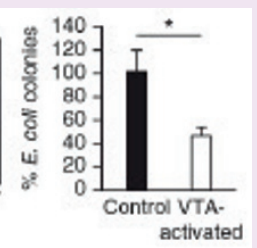

challenge of the mice with exposure to bacteria, they demonstrated an increase in innate and adaptive immune responses. Having determined that the remaining bacterial load was significantly reduced in VTA-activated mice compared with the control group, they investigated the source of the immune activation. Because dopamine does not cross the blood-brain barrier, they hypothesized involvement of the sympathetic nervous system. Although more experimentation will be needed to more accurately identify the effects of naturally occurring reward responses on immunity, these data suggest a pathway for the placebo effect and show a causal relationship between the brain's reward system and immunity.

Nature Medicine 2016;22:940-944; doi:10.1038/nm.4133

\section{Fibronectin required for regeneration of skeletal muscle}

Several factors may contribute to the agerelated changes in skeletal muscle stem cells (MuSCs). Lukjanenko et al used fibronectin (FN) knockout models to demonstrate that regenerating muscle replicated the aging phenotype, and they observed a decrease in MuSC numbers. Using an antibody array, mouse MuSC-derived myoblasts were grown on FN for 72 hours and compared with those grown on collagen I. Sixty-four

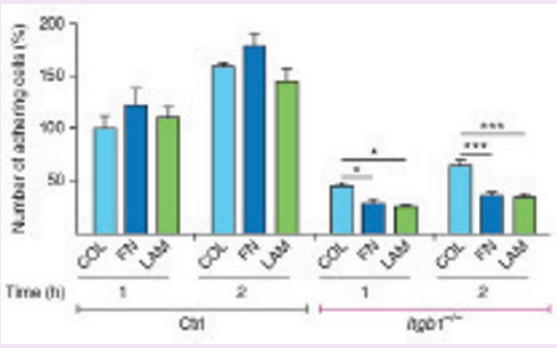
of 1,318 site-specific antibodies showed differing phosphorylation levels between the two groups-notably, $\beta 1$-integrin and other components involved in ERK, MAPK, and cell-cycle regulatory pathways and also known to be altered as a consequence of aging. The investigators then found that aged MuSCs have a reduced adhesive capacity and that FN treatment could rescue this loss of capacity. By implicating a candidate protein as a root cause of MuSC aging, this group has identified a potential target for rejuvenating muscle tissue in our aging society. Nature Medicine 2016;22:897-905; doi:10.1038/nm.4126

\section{GME population variome to characterize diversity}

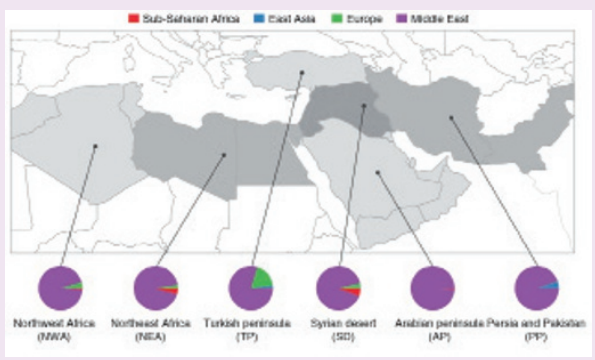

To investigate recessive disease, Scott et al generated a whole-exome Greater Middle East (GME) variome from 1,111 unrelated subjects. The area was selected as a site of human migration and population admixture. The group demonstrated that there was wide diversity and high inbreeding coefficients in GME population substructure. This contrasted with unique genetic variation represented in GME

populations. The findings supported records of migration and conquests and revealed a previously unstudied GME component. Given this population with millennia of elevated rates of consanguinity, there had been an expectation of purging of recessive alleles. Instead, the authors found large, rare, homozygous blocks that supported the occurrence of recent consanguineous matings. This enabled them to identify genes harboring putatively highimpact homozygous variants in healthy humans from the population. The data, which are publicly accessible, will facilitate other genomic studies in global and GME populations. Nature Genetics, published online 18 July 2016; doi:10.1038/ng.3592

Emma Judson contributed to these reviews. 